\title{
O uso de vidro reciclado na confecção de membranas para microfiltração
}

\section{(The use of recycle glass in microfiltration membrane processing)}

\author{
O. B.G. Assis \\ Embrapa Instrumentação Agropecuária \\ Rua XV de Novembro, 145, C.P. 741 \\ S. Carlos, SP 13560-970 \\ odilio@cnpdia.embrapa.br
}

\begin{abstract}
Resumo
Peças sinterizadas porosas para uso como meio de filtragem foram produzidas a partir de pós de vidro oriundo de material reciclado. O processamento seguiu o princípio de preenchimento, no qual sal $(\mathrm{NaCl})$ foi adicionado como fase não sinterizável, solúvel, com o objetivo de controle dos tamanhos de poros e da porosidade. Detalhes da moagem e características dos pós processados são apresentados. A microestrutura das membranas sinterizadas e lixiviadas foi caracterizada por microscopia eletrônica de varredura e porosimetria de mercúrio. Os resultados indicam dependência entre a fração de sal adicionada e a microestrutura. Poros resultantes da solubilização do sal são identificados por apresentarem formatos preferencialmente regulares, diferindo dos demais. Análises de permeabilidade por queda de pressão demonstram a possibilidade de operação em microfiltração para diferentes intervalos de tamanho de poros.

Palavras-chave: vidro reciclado, processamento de membranas porosas, principio do preenchimento, microfiltração.
\end{abstract}

\begin{abstract}
Open-pore sintered pieces were produced from waste glass powder, as raw material. The processing followed the filler principle, by making use of a salt ( $\mathrm{NaCl}$ ) mixed with glass powder, as soluble, non-sinterizable phase aiming at a control of porous size and porosity. Milling details and powder features are presented. The microstructure of the sintered and washed compacts was characterized using SEM and mercury porosimetry. The results showed that the final density depends strongly on the salt volume fraction. Salt dissolution generates pores with regular morphology predominating flat. Pressure-decay permeability analysis indicates the possibility of microfiltration operation in different ranges of porous size.
\end{abstract}

Keywords: recycled glass, porous membranes processing, filler principle, microfiltration.

\section{INTRODUÇÃO}

O montante de vidro descartado (garrafas, vasilhames diversos, vidros planos da construção civil, etc.) representa, em nível mundial, algo em torno de 7,5\% em peso do total de lixo doméstico gerado. Para se ter uma idéia numérica dessa quantidade, os EUA consumem em média um volume de embalagens de vidro superior a $30 \mathrm{Kg} / \mathrm{habitante} / \mathrm{ano}$ [1] e no Brasil esses valores são estimados em 5,57 kg per capita por ano [2], o que de uma forma geral traduz em milhares de toneladas de material vítreo diariamente rejeitados e acumulados como lixo. Evidentemente este tem se tornado um sério problema tanto do ponto de vista financeiro quanto ambiental, para qualquer país industrializado ou em desenvolvimento.

A este cenário soma-se que a reutilização do vidro, ou de qualquer outro material passível de reciclagem, ainda não é um empreendimento considerado vantajoso.

Embora pesquisas e avanços técnicos sejam constantes na direção de transformar e reciclar vidros usados em produtos úteis e de interesse comercial, pouco tem sido conseguido na prática além da transformação dos vasilhames descartados em novos vasilhames de qualidade igual ou inferior, ou em fibras para uso como isolantes ou para preenchimentos diversos. A manutenção das características originais de um vidro é sem dúvida o gargalho da reciclagem. A mistura de composições altera as características físicas do material e, principalmente para os vidros coloridos, a variedade de padrões gera tons indefinidos e não reprodutíveis [3].

O grande atrativo na reciclagem do vidro recai na economia energética e de matéria prima para o produtor. De uma forma geral, $55 \%$ dos custos de produção vêem da aquisição de matéria prima, 30 \% de mão de obra e $15 \%$ da energia. Segundos dados da CEMPRE [4], cada quilo de vidro reciclado na forma moída substitui 6,6 quilogramas de areia e a refusão de 1 tonelada consome em média 70\% menos energia do que a requerida para a fabricação inicial. Além do mais, vidros produzidos com material reciclado reduzem a quantidade de emissão de poluentes no ar em cerca de $20 \%$ e na água na ordem de $50 \%$. 
O Brasil reciclou em 2001, 42\% das 883 mil toneladas de vidro para embalagens produzidas no país em novas embalagens, sendo o Estado de São Paulo responsável por $40 \%$ do total reciclado [5]. O que não deixa de ser um dado significativo considerando que a média de reciclagem na Europa não é superior a 50\% e o Japão reaproveita 55,5\% do vidro que consome [6]. Contudo, é consideravelmente inferior ao avaliarmos isoladamente países como a Suíça e a Áustria, com 91 e 88\% de reciclagem respectivamente [7].

Além da reconfecção de vasilhames ou similares, existe uma série de possibilidades para o uso ou incorporação de vidro reciclado em produtos de cunho tecnológico ou em processos de engenharia avançada.

O trabalho pioneiro de Brown e MacKenzie em 1982 [8] demonstrou o uso satisfatório de vidro reciclado, na formação de cerâmicas industriais com elevada resistência “verde”, além da possibilidade da vitrificação superficial. Hoje a vitrificarão de azulejos, pisos e telhas com material reciclado já têm sido uma prática corrente $[9,10]$. Vidros moídos também podem ser empregados como abrasivos de uso industrial, em substituição ao jato de areia, com boa eficiência em função do formato angular típico de suas partículas.

Estudos mais recentes tem avaliado o uso de PGA (pulverized glass aggregate), como substituto ao cascalho na pavimentação asfáltica $[1,11,12]$. Tem-se observado contudo, que adições superiores a $10 \%$, embora elevem a durabilidade do pavimento, apresenta destacamentos progressivos e ações abrasivas, em longo prazo, nos pneus automotivos [13]. PGA também tem sido amplamente estudado como aditivo em concretos de usos na construção civil [14]. O chamado Glascrete [15] é um produto que apresenta adições em até $30 \%$ de vidro britado ao cimento com o objetivo de elevar as propriedades mecânicas. Segundo análises realizadas por Shao et al. [16], há um incremento significante na resistência ao impacto e compressão nos concretos reforçados com matrizes vítreas. Nesse sentido, deve-se ter em mente a possibilidade de reações alcalinas da sílica, que pode vir a enfraquecer a estrutura ao longo do tempo. Berube [17] demonstrou, contudo, que essas reações são controláveis e estão ausente em misturas com pós finos de vidro $(<2-3 \mathrm{~mm})$.

Produtos mais elaborados como a confeç̧ão de peças refratárias a base de vidro reciclado [18], compósitos vítreos sinterizados, de elevada resistência termo-mecânica [19] ou porcelanas domésticas para microondas, aonde adições de vidro reciclado elevam a eficiência de aquecimento [20], são alguns dos produtos em desenvolvimento que fazem uso de vidro descartado.

Vidros moídos têm também grande potencial para aplicações na área ambiental. O uso de material fragmentado como substrato para sustentação de raízes e meio de retenção de água em sistemas de hidroponia, foi avaliado com resultados similares aos obtidos para a argila [21], e em sistemas de purificação em larga escala, como filtração lenta, como uso alternativo à areia $[22,23]$. Este é um mercado a ser plenamente explorado, considerando que os sistemas públicos de tratamento de água repõem uma média de 750.000 toneladas de areia ao ano. O vidro além de ser $20 \%$ mais leve que a areia, apresenta um melhor empacotamento em função do formato angular de suas partículas, o que gera uma retenção mais eficiente sem perdas na permeabilidade [24], e recentemente avaliamos o emprego de pós de vidro reciclado como matéria prima na confecção de filtros e suportes para a imobilização de enzimas, seja para uso em reatores catalíticos ou em sensores enzimáticos [25, 26].

A possibilidade de alterações das características físicoquímicas superficiais como a modificação ou implante de grupos funcionais; a maximização da hidrofilicidade ou mesmo a mudança para um comportamento hidrofóbico, conseguido por tratamentos químicos relativamente simples, torna o vidro definitivamente um material de interesse para a confecção de dispositivos de interação superficial, como membranas ou substratos ativos para uso em microfiltração.

A unidade da Embrapa Instrumentação Agropecuária em S. Carlos, SP, já há algum tempo tem avaliado potenciais usos para o vidro reciclado, principalmente na confecção de dispositivos de pequeno porte para filtragem de água, de baixo custo e de fácil confecção, para uso geral e em particular na zona rural $[27,28]$.

Neste trabalho caracterizamos a estrutura resultante do emprego de vidros reciclados como matéria prima para o processamento de elementos porosos sinterizados para uso em microfiltração.

\section{EXPERIMENTAL}

\section{Produção e caracterização dos pós de vidro}

O material de partida constituiu de garrafas transparentes, de uma mesma origem, cuja composição química média, expressa em massa é $\mathrm{SiO}_{2}$ (72,8\%); $\mathrm{Na}_{2} \mathrm{O}(13,2 \%) ; \mathrm{CaO}(11,2 \%) ; \mathrm{MgO}$ (0,16\%); $\mathrm{Al}_{2} \mathrm{O}_{3}$ (2,13\%); $\mathrm{FeO} / \mathrm{Fe}_{2} \mathrm{O}_{3}(0,039 \%) ; \mathrm{K}_{2} \mathrm{O}(0,09 \%)$, segundo determinação da Saint-Gobain (Centro Técnico de Elaboração do Vidro). As garrafas foram previamente lavadas, secas em estufa e manualmente fragmentadas em partículas da ordem de 1,5 cm. Esse material foi em seguida reduzido em moinho de bolas de alumina, em rotação de 36 rpm, em cargas individuais de $200 \mathrm{~g}$. Amostras dos pós foram coletadas em intervalos de $2 \mathrm{~h}$, até um total de $12 \mathrm{~h}$, para acompanhamento das dimensões e formato.

A morfologia foi avaliada por microscopia eletrônica de varredura e a distribuição de tamanhos caracterizada por sistema de medida granulométrica por atenuação de raios gama, em metodologia estabelecida e previamente descrita [29, 30].

\section{Processamento das membranas}

O processamento das membranas se deu pela técnica do preenchimento (filler principle), seguindo procedimento apresentado por Siebers e co-autores [31], no qual uma fase 
solúvel em água, não-sinterizável, é adicionada ao pó de vidro anterior a etapa de sinterização, cuja remoção posterior por lavagem contribui para o controle da estrutura porosa.

Para a confecção das membranas, pós de tamanho médio próximo a $50 \mu \mathrm{m}$ foram empregados. $\mathrm{NaCl}$ (PA-Synth) foi adicionado como fase solúvel. Avaliaram-se adições de sal nas proporções de 10,15, 20, 25, 30 e $40 \%$ em peso. O sal foi peneirado em dimensões da ordem de $20 \mu \mathrm{m}$ anterior a mistura.

A compactação se deu para porções próximas a $6 \mathrm{~g}$ de mistura, em matriz metálica cilíndrica com diâmetro interno de $32 \mathrm{~mm}$, para pressão uniaxial de $120 \mathrm{MPa}$. Os corpos finais apresentaram altura de $3,5 \mathrm{~mm}$. A sinterização foi conduzida na temperatura de $750{ }^{\circ} \mathrm{C}$ no seguinte ciclo térmico: taxa de aquecimento de $10{ }^{\circ} \mathrm{C} /$ min e manutenção em patamar de sinterização por uma hora seguido de resfriamento no forno. O processo ocorreu sem controle de atmosfera. Após completo resfriamento as peças eram levadas em água corrente por $24 \mathrm{~h}$. A Fig. 1 esquematiza o ciclo completo do principio de preenchimento.

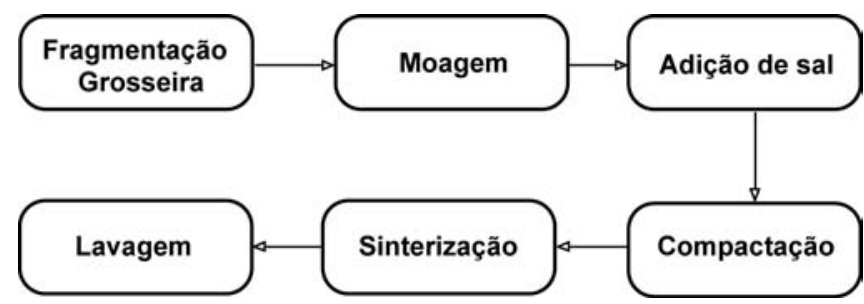

Figura 1: Etapas do processamento pela técnica do preenchimento. [Figure 1: Sequence of filler principle processing.]

\section{Caracterização}

A caracterização das peças sinterizadas seguiu procedimentos padrões como observação por microscopia eletrônica por varredura (Philips XL 30), porosimetria por intrusão de mercúrio (Pore Sizer 9320, Micrometrics) e de medidas de permeabilidade em sistema de queda de pressão em câmara cilíndrica com fluxo perpendicular a amostra, segundo montagem já descrita [32, 33]. As medidas foram realizadas na temperatura ambiente por passagem de ar sob pressão. A densidade do ar foi considerada como $\rho=$ $1,079 \mathrm{~kg} / \mathrm{m}^{3}$ e a viscosidade $\mu=1,83 \times 10^{-5}$ Pa.s. Os testes foram realizados em duplicatas para garantir repetitividade. Os dados foram calculados tendo por base os conceitos de permeabilidade Darciana [34].

\section{RESULTADOS E DISCUSSÃO}

A Fig. 2 apresenta a evolução de tamanhos de partículas em função do tempo de moagem. Observa-se que para as primeiras $4 \mathrm{~h}$ a redução na dimensão média das partículas não é superior a 30\% com respeito ao tamanho inicial, contudo a distribuição de tamanhos é estreitada, o que pode ser observada pela diminuição do desvio em cada medida. Após esse período, a cominuição torna-se mais expressiva com uma rápida diminuição tanto nas dimensões como no desvio. Este aspecto é melhor observado ao compararmos, por exemplo, as curvas de distribuição para amostras moídas em 2 e $4 \mathrm{~h}$, cujos tamanhos médios são bastante próximos (99,43 $\pm 32 \mu \mathrm{m}$ e $80,52 \pm 22,57 \mu \mathrm{m}$, respectivamente). A distribuição segue um perfil do tipo log-normal, onde tem-se o estreitamento do espectro com ligeiro acréscimo para uma freqüência para menores dimensões.

A Fig. 4 apresenta o aspecto dos pós, em amostra de $2 \mathrm{~h}$

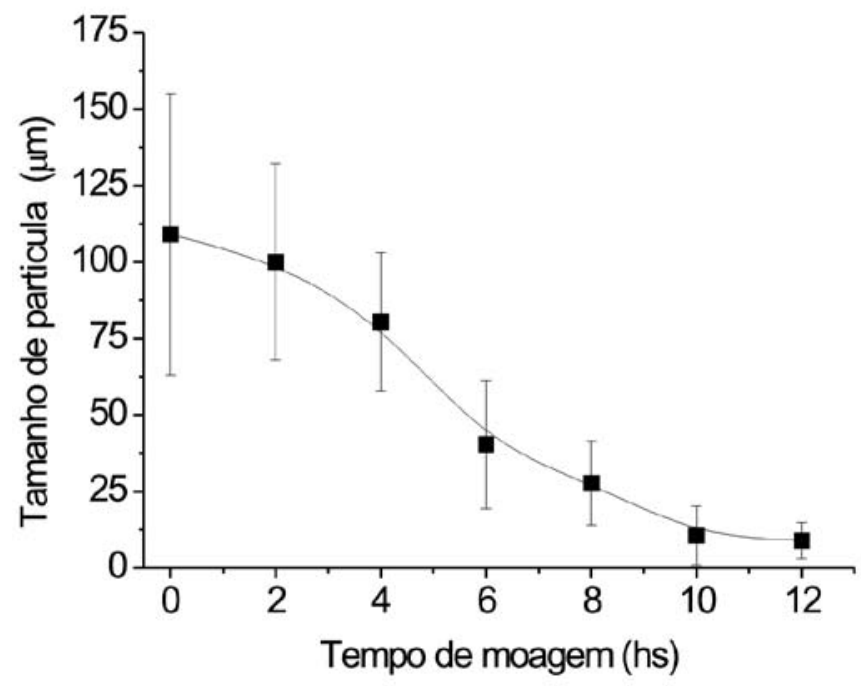

Figura 2: Evolução do tamanho de partículas em função do tempo de moagem.

[Figure 2: Particle size evolution as a function of milling time.]

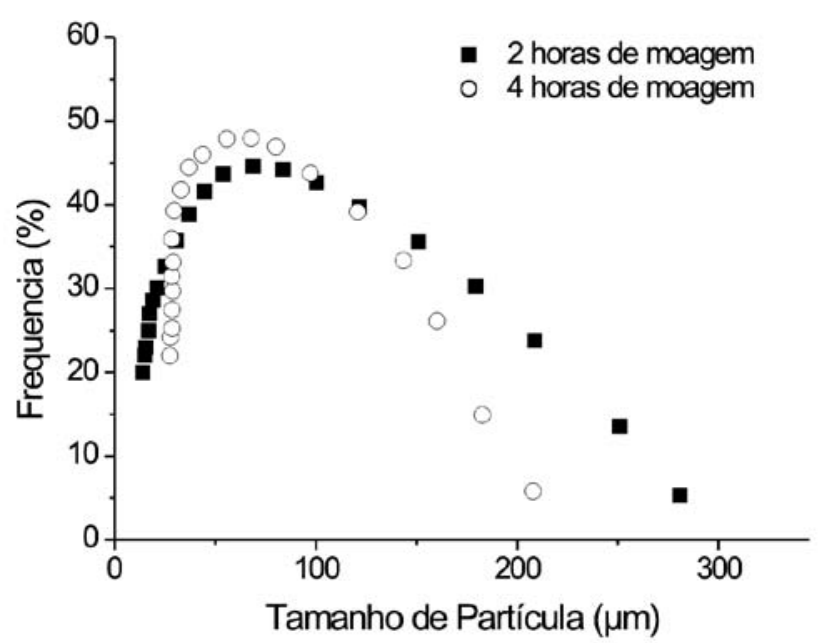

Figura 3: Exemplos de distribuição de tamanhos de partículas para moagens em 2 e 4 horas.

[Figure 3: Examples of particle size distributions for 2 and 4 hours milling.] 
de moagem, por microscopia eletrônica de varredura, sendo possível visualizar a distribuição de formatos e tamanhos gerados no período.

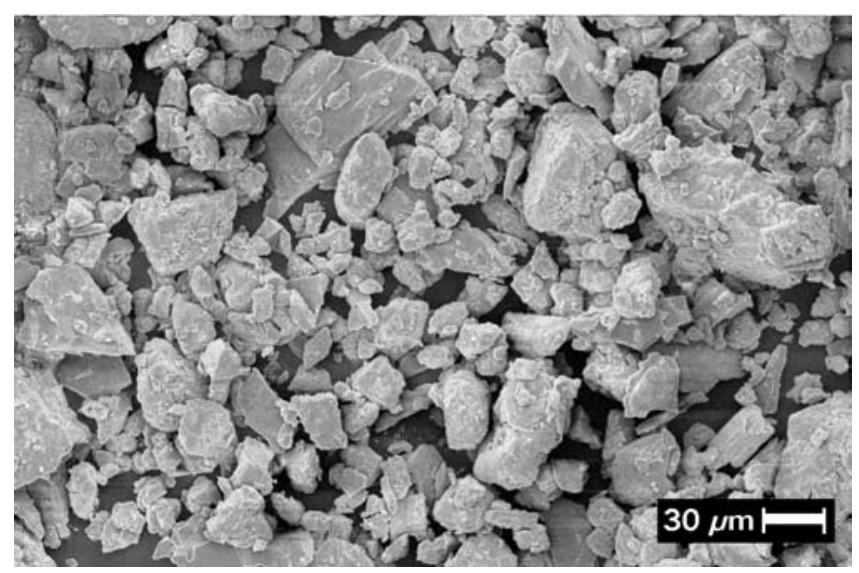

Figura 4: Aspecto do pó obtido após 2 horas de moagem.

[Figure 4: Glass powder after 2 hours milling.]

Por ser o vidro um material tipicamente frágil, esperava-se inicialmente que o processo de moagem por bolas resultasse em uma redução rápida e homogênea dos fragmentos, o que não foi plenamente observado. Avaliações mais rigorosas indicaram que as dimensões iniciais e volume de carga no moinho, evidentemente são os fatores determinantes na eficiência de moagem e devem ser levados em consideração na obtenção de pós de vidro com as características desejadas [27, 33]. A condição ideal almejada é de partículas com dimensões inferiores a $40 \mu \mathrm{m}$ em um perfil de distribuição estreita de tamanhos, o que permitirá um melhor controle do processo e redução das variáveis de sinterização.

Independente das dimensões dos pós, a sinterização de vidros sodo-cálcicos ocorre como conseqüência de fluxo viscoso para temperaturas entre $600-800{ }^{\circ} \mathrm{C}$, segundo mecanismos detalhados por Scherer [35] e Chiang et al. [36]. Ao atingir a temperatura de amolecimento, em torno de 536 - $696{ }^{\circ} \mathrm{C}$ [37], tem-se o inicio do processo de coalescimento das partículas. Nessas condições, os pescoços inicialmente estabelecidos apresentam raios de curvaturas menores aos comparados com a curvatura da superfície das partículas, o que resulta em uma pressão negativa em direção ao pescoço. Esse gradiente de pressão precipita o movimento viscoso para a região interpartículas, com rápido deslocamento de massa. Uma vez iniciado este fluxo há pouco controle externo sobre a estrutura final resultante, em função da velocidade do processo. Para a manufatura de estruturas porosas com certo controle, a adição de fase não sinterizável tornase um recurso necessário. Nestas condições há uma tendência de fluxo e acomodação de matéria ao redor dos grãos de sal envolvendo suas faces. Se o processo for interrompido oportunamente (anterior a um completo encapsulamento do sal) e com a subseqüente lixiviação, os poros resultantes assumirão morfologia característica da fase solúvel. A Fig. 5 ilustra esquematicamente a suposta formação de poros por este principio.

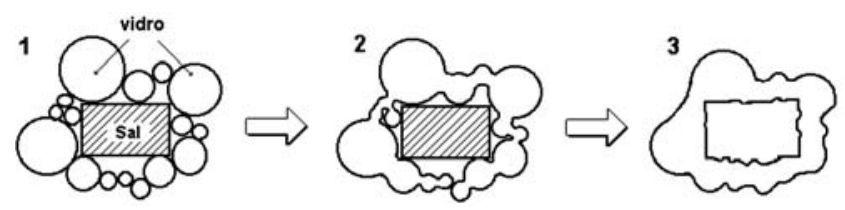

Figura 5: Ilustração esquemática do mecanismo de formação de poros pela técnica do preenchimento: em (1) compacto com presença do sal; (2) ao longo do aquecimento e (3) após a remoção do sal.

[Figure 5: Scheme of the pore formation by viscous sintering, according to the "filler principle" technique. (1) is the green compact, composed by glass powder and salt particles (grey); In (2) neck formation between neighbor glass particles, and (3) a pore surrounding a previous salt particle, which has been washed out.]

Na Fig. 6 temos como exemplo a curva de densificação em função do tempo de aquecimento, a partir da temperatura ambiente, para uma membrana processada com adições de $20 \%$ de $\mathrm{NaCl}$. A densidade da peça, atinge um máximo experimental logo nos primeiros minutos no patamar de sinterização (intervalo definido em $750{ }^{\circ} \mathrm{C}$ ), indicando a rapidez do processo no mecanismo de fluxo viscoso. Esses valores reafirmam o difícil controle, por parâmetros externos, de uma estrutura definida na ausência de fase controladora de poros. Este comportamento é similar e representativo para as demais granulometrias e adições avaliadas.

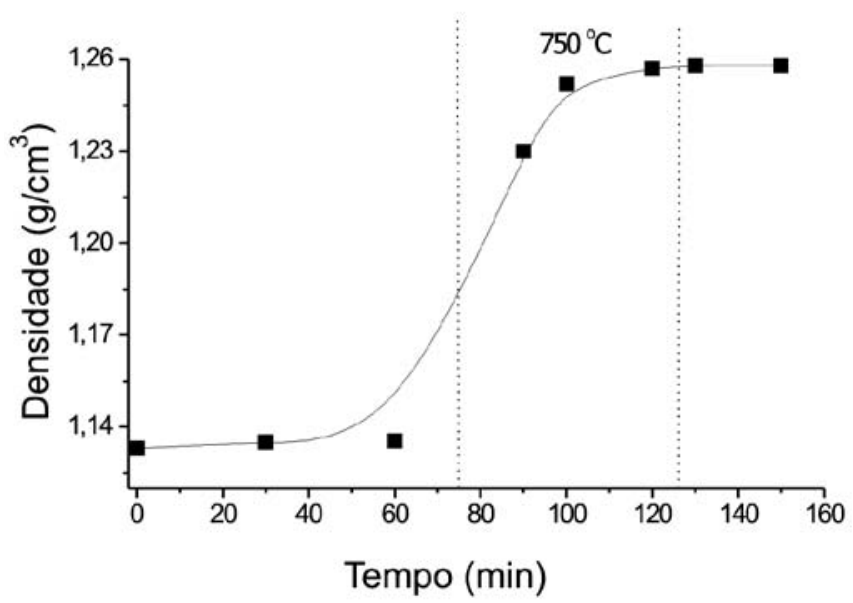

Figura 6: Curva da densidade para a membrana processada com $20 \%$ de $\mathrm{NaCl}$ (aquecimento $10{ }^{\circ} \mathrm{C} / \mathrm{min}$ a partir da temperatura ambiente).

[Figure 6: Densification curve of a $20 \% \mathrm{NaCl}$ membrane (heating at $10^{\circ} \mathrm{C} / \mathrm{min}$ starting at room temperature).]

A Fig. 7 apresenta o aspecto superficial e interno (superfície fraturada) de amostra processada com 20\% de $\mathrm{NaCl}$, indicando uma estrutura composta por morfologias distintas. As superfícies das membranas de um modo geral 
apresentam considerável irregularidade estrutural, que pode ser atribuída a fatores como uma pior distribuição de sal nessa região associada a um empacotamento irregular dos pós em função da ação imediata da pressão de compactação. A estrutura torna-se mais homogênea no interior da peça.
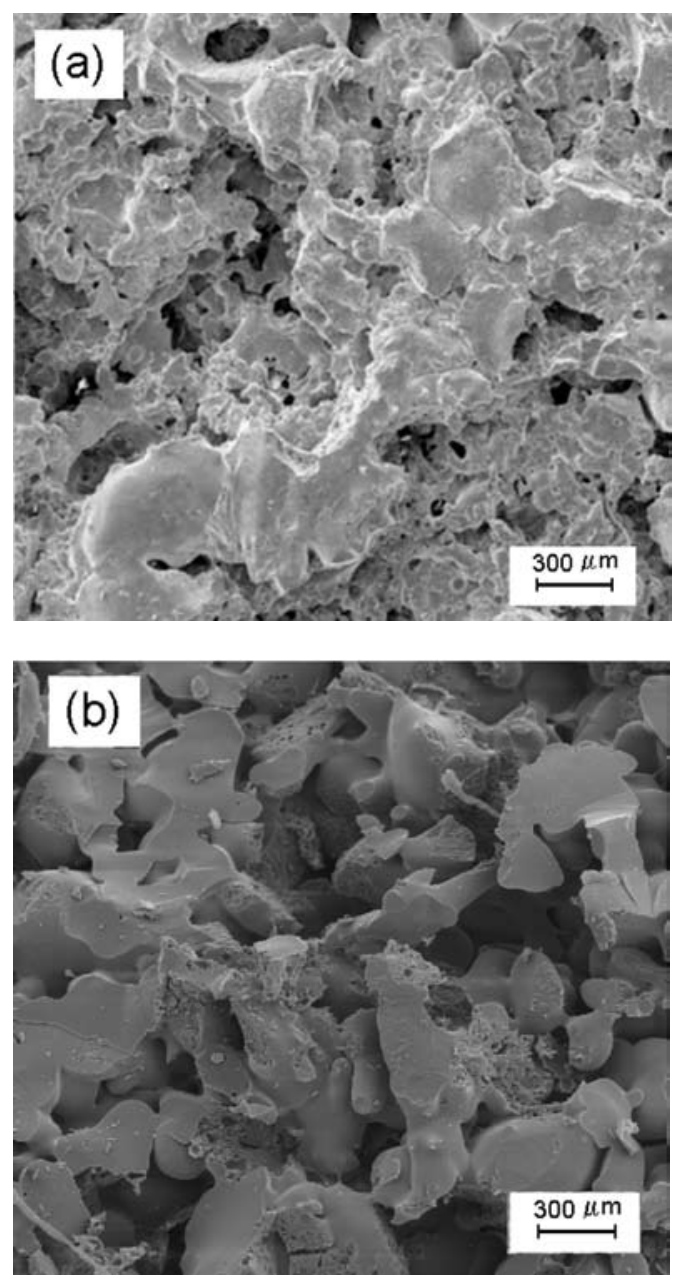

Figura 7: Aspecto estrutural da superfície (a) e região interna (b), superfície de fratura de membrana sinterizada com $20 \%$ de $\mathrm{NaCl}$. [Figure 7: Aspects of surface (a) and internal region (b), fractured surface, of a $20 \% \mathrm{NaCl}$ sintered membrane.]

A distribuição e a conectividade entre os poros são fatores determinantes em processos de filtragem. Mesmo com o uso da técnica do preenchimento espera-se encontrar na estrutura final uma combinação de poros formados por mecanismos diversos, ou seja, poros resultantes da interrupção térmica, sem influência do sal; poros formados pela retenção de gases ou por estágio final de sinterização, que geralmente são isolados e de formato esféricos e os poros decorrentes do envolvimento e solubilização dos grãos do sal. Essas estruturas são caracterizadas nas observações microscópicas onde temos poros não conectados encontrados preferencialmente nas regiões periféricas das peças e poros abertos no interior.
Uma análise mais detalhada e seletiva dos poros que constituem a maioria da estrutura sinterizada, confirma o modelo da Fig. 5. A solubilização do sal após a sinterização provoca poros que são moldados, predominantemente, pelos formatos dos grãos de sal. $\mathrm{O} \mathrm{NaCl}$ possui rede primária cúbica sendo que grãos e aglomerados tendem a apresentar empacotamentos com faces planas e paralelas. A Fig. 8 exemplifica o aspecto dos poros nitidamente gerados pela solubilização do sal. Estudos complementares mostraram que sais de composições diferentes alteram a morfologia dos poros [26, 38].

A formação de poros com alta conectividade e com paredes tendendo a superfícies planas vai de encontro ao potencial uso dessas membranas. Uma condição hipotética, pode ser visualizada em uma distribuição aleatória de poros regulares em uma direção, assumindo considerações de percolação apresentadas por Thovert et al. [39], Fig. 9.

O aumento da tortuosidade nesta condição otimiza possíveis interações entre o permeado e a superfície dos poros, pelo aumento do "tempo de residência" do fluido na estrutura. Como a opção para o emprego de filtros de vidros se dá principalmente por sua capacidade de interação superficial, seja como substrato para formação de filmes biologicamente ativos, e posterior ação como biofiltro, ou para adsorção de contaminantes e resíduos químicos diretamente em sua superfície [25, 38], sua ação fica assim potencializada.

A maioria dos poros na estrutura que não foram gerados pela presença de sal é de fácil identificação e apresentam um formato distinto. A Fig. 10 exemplifica alguns desses poros. Embora contribuam para a medida de porosidade volumétrica, não apresentam características ideais de filtragens como as conseguidas pelos gerados pelo princípio do preenchimento.

Em princípio, a técnica do preenchimento tem como intuito estabelecer uma relação entre a quantidade de sal adicionada e a porosidade gerada. Há evidentemente um limite máximo de adição a partir do qual é sensivelmente reduzido o grau de sinterabilidade com ocorrência de perda da resistência mecânica [33]. Pelo conjunto de medidas obtidas por porosimetria observa-se comportamento similar entre o teor de sal adicionado e a porosidade e os tamanhos médios dos poros resultantes após lixiviação (Fig. 11). Para uma resposta inicialmente linear, a curva indica que para teores de sal acima de $25 \%$, temos reduzido a influencia sobre a porosidade final. As medidas de perda de massa expressas na Tabela I demonstram que a remoção do sal após a sinterização por solubilização é quase total. Na pratica a porosidade obtida corresponde, a valores superiores (acima de 30\% de massa adicionada) ao da fração volumétrica adicionada e removida (Fig. 11), indicando claramente a contribuição de estrutura porosa não relacionada à presença de sal.

O estabelecimento de uma estrutura de poros interconectados em maior ou menor dimensão e em variação volumétrica interfere no escoamento do fluído 

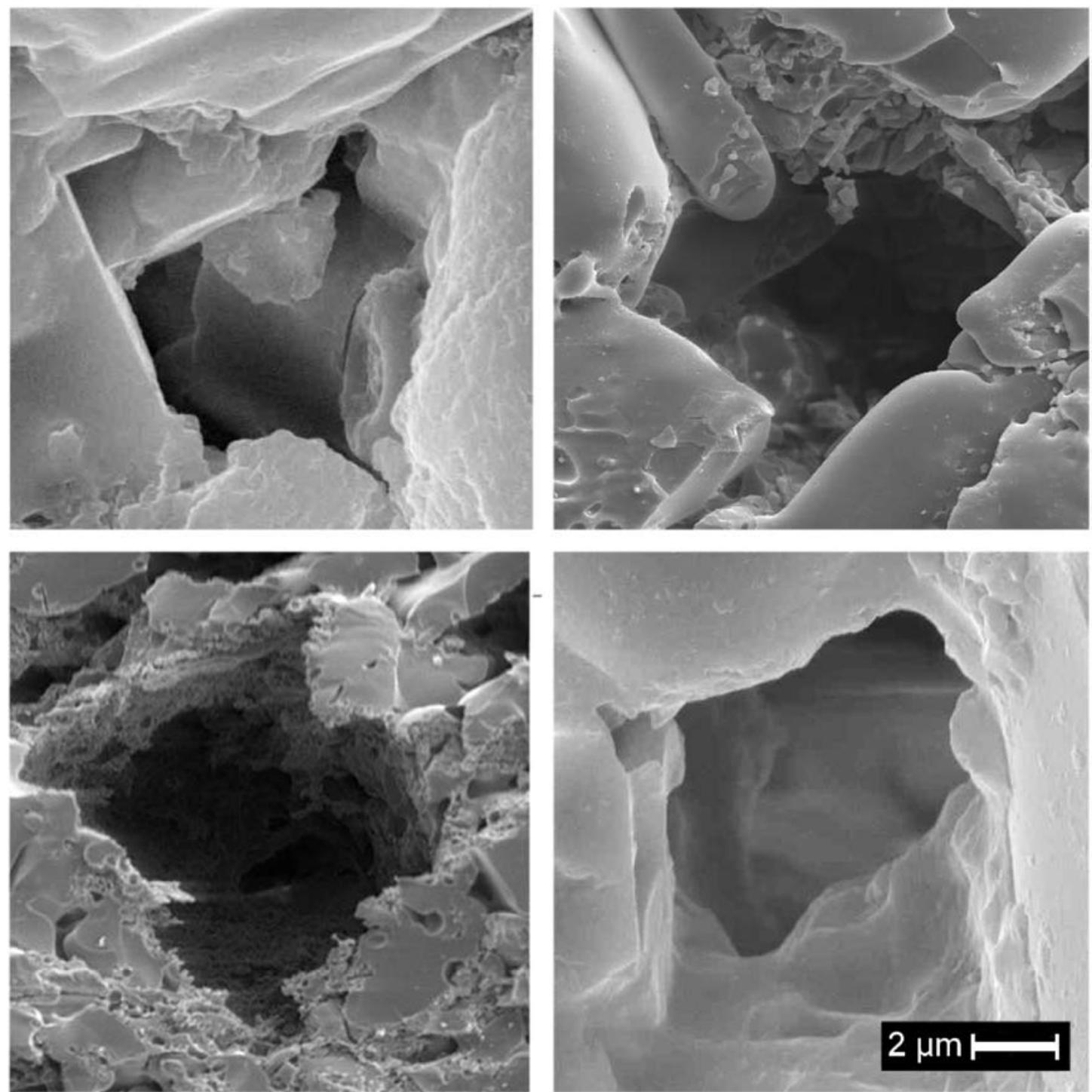

Figura 8: Exemplo da morfologia predominante de poros, decorrentes da remoção do $\mathrm{NaCl}$ da estrutura sinterizada em uma mesma amostra (adição de $30 \% \mathrm{NaCl}$ ).

[Figure 8: Examples of predominant porous morphology due to $\mathrm{NaCl}$ removal from sintered structure found in a same sample (30\% $\mathrm{NaCl}$ added).]

alterando as características de filtragem. Uma maior tortuosidade, associada a uma menor porosidade geram redução na permeabilidade. Essa característica pode ser inferida através das curvas de queda de pressão tomadas nas diversas condições de sinterização, expressas na Fig. 12. Por parâmetro de pressão entende-se a razão entre as pressões medidas anterior e posterior à passagem pelo meio poroso, segundo a relação:

$$
\frac{\mathrm{P}_{\mathrm{f}}^{2}-\mathrm{P}_{0}^{2}}{2 \times \mathrm{P}_{\mathrm{atm}} \times \mathrm{L}}
$$

sendo L a espessura da membrana, $\mathrm{P}_{\text {atm }}$ a pressão atmosférica local e $\mathrm{P}_{0}$ e $\mathrm{P}_{\mathrm{f}}$, respectivamente, a pressão anterior e posterior à passagem pela membrana. Menores valores deste parâmetro indicam uma maior permeabilidade e vice-versa [33, 40]. Assim, os resultados obtidos estão em concordância com o esperado, tendo por base as características microestruturais, Ou seja, o parâmetro é sensivelmente reduzido nas amostras com maiores tamanhos de poros e de porosidade volumétrica. O que significa que a queda de pressão é reduzida para concentrações de sal acima de $20 \%$ reafirmando uma maior permeabilidade para membranas processadas a partir dessas condições.

Esse resultado é de relevancia prática ao considerarmos que a permeabilidade comporta-se relativamente similar para um certo intervalo de dimensão de poros e porosidade. A quase totalidade do uso de filtros tem por princípio a 

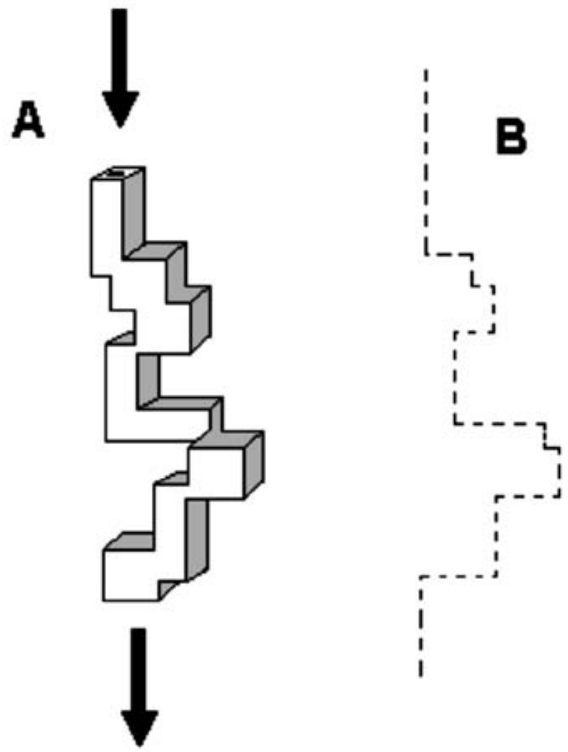

Figura 9: Condição hipotética da distribuição de poros com faces paralelas aleatoriamente distribuídos na estrutura (A). Ilustrando o aumento do caminho devido à tortuosidade, proporciona um maior tempo de residência do permeado na estrutura (B).

[Figure 9: Hypothetical condition of flat faced boxes randomly place one over the other (A) illustrate the increase of the liquid path in one direction $(B)$ inside the porous material, increasing the permeated resident time.]

filtragem por "peneiramento", ou seja, a contenção fracionada ou contínua de partículas ou colóides em suspensão pela interrupção de sua passagem por orifício de menor diâmetro que suas dimensões.

Considerando o que o processo aqui empregado, de preenchimento, permite obter membranas com tamanhos de poros diversos, sem alteração significativa da permeabilidade, eleva o potencial de aplicação dessas peças para uso seletivo na retenção específica de contaminantes de determinados intervalos de dimensão em meio líquido.

Outro aspecto de importância a ser observado diz respeito a possíveis reações que podem ocorrer na
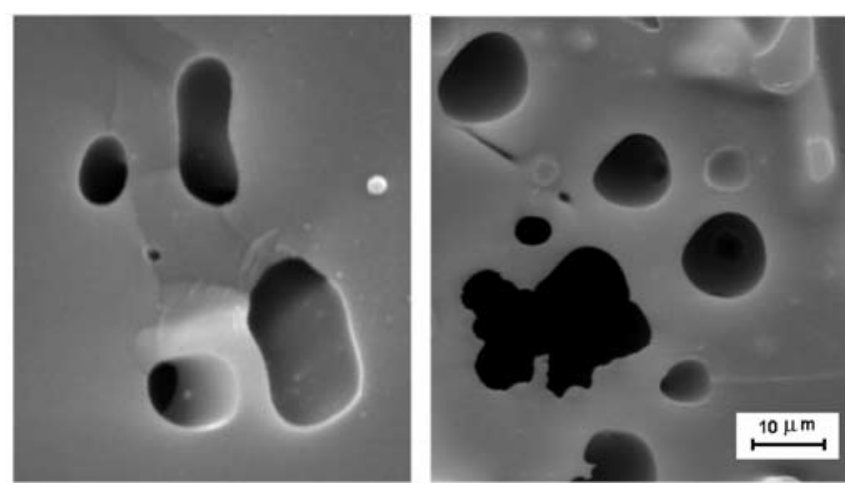

Figura 10. Aspecto de poros formados na estrutura vítrea sem a presença de sal, diferindo dos gerados pela solubilização do $\mathrm{NaCl}$ (amostra com 30\% NaCl).

[Figure 10: Aspect of formed porous into vitreous structure without salt effect, differing from those obtained by solubilization (sample with 30\% $\mathrm{NaCl}$ ).]

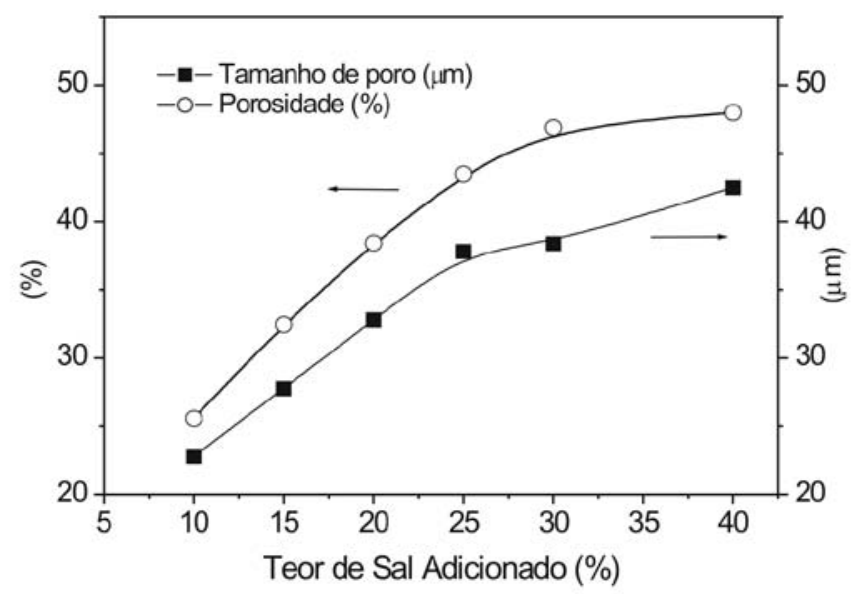

Figura 11: Influência do teor de sal na porosidade volumétrica e no tamanho médio final de poros na estrutura sinterizada.

[Figure 11: Influence of added salt on the volumetric porosity and on average porous size in sintered structure.]

Tabela I - Medidas de perda de massa anterior e após solubilização do sal.

[Table I - Mass loss before and after slat solubilization.]

\begin{tabular}{ccccc}
\hline $\begin{array}{c}\text { Teor de NaCl } \\
\text { Adicionado } \\
\text { (\% em peso) }\end{array}$ & $\begin{array}{c}\text { Massa } \\
\text { correspondente } \\
\text { de NaCl (g) }\end{array}$ & $\begin{array}{c}\text { Massa do } \\
\text { sinterizado } \\
\text { (vidro + sal)(g) }\end{array}$ & $\begin{array}{c}\text { Após } \\
\text { solubilização } \\
\text { (g) }\end{array}$ & $\begin{array}{c}\text { Fração } \\
\text { estimada de } \\
\text { sal removido (\%) }\end{array}$ \\
\hline 10 & 0,60 & 5,51 & 4,92 & 98,3 \\
15 & 0,90 & 5,57 & 4,69 & 97,7 \\
20 & 1,20 & 5,52 & 4,34 & 98,3 \\
25 & 1,50 & 5,57 & 4,10 & 98,0 \\
30 & 1,80 & 5,58 & 3,80 & 98,8 \\
40 & 2,50 & 6,42 & 3,96 & 98,4 \\
\hline
\end{tabular}


superfície dos vidros de soda-cal na presença de meio aquoso, principalmente quando as membranas estiverem em operação. As fases $\mathrm{Na}_{2} \mathrm{O}$ e $\mathrm{K}_{2} \mathrm{O}$ são solúveis em água, favorecendo a ocorrência de ataque primário, ou seja, de trocas entre íons sódio $\left(\mathrm{Na}^{+}\right)$e potássio $\left(\mathrm{K}^{+}\right)$do vidro e íons hidrogênio da água, o que pode alterar a estrutura e características superficiais dos poros. Segundo Sinton

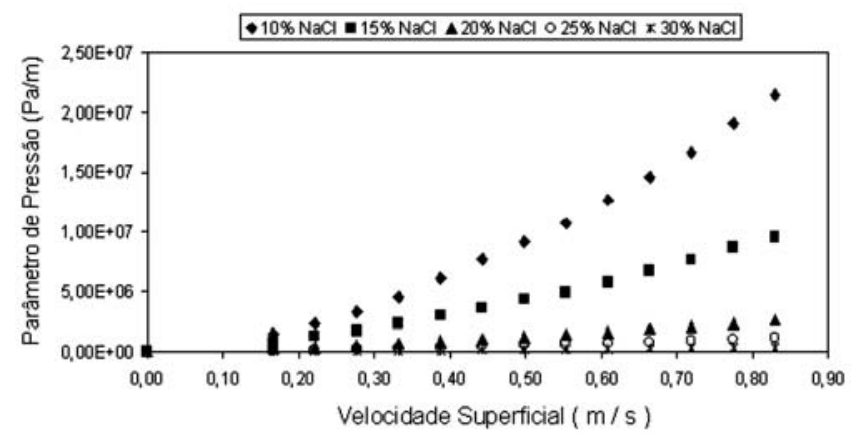

Figura 12: Curvas experimentais da queda de pressão através das amostras sinterizadas para diversas concentrações de sal, como fase geradora de poros.

[Figure 12: Pressure-drop experimental curves through sintered samples at several salt concentrations.]

e LaCourse [41], a cinética de dissolução dessas fases é fortemente dependente do $\mathrm{pH}$ do meio interativo. Para um meio inicialmente neutro, a reação acarretaria em um emprobrecimento, por lixiviação, dessas fases alcalinas, segundo uma reação simples do tipo:

$$
(\mathrm{Si}-\mathrm{O}-\mathrm{R})_{\text {vidro }}+\mathrm{H}_{2} \mathrm{O} \rightarrow(\mathrm{Si}-\mathrm{O}-\mathrm{H})_{\text {vidro }}+\mathrm{R}^{+}+\mathrm{OH}_{\text {solução }}^{-}
$$

o que geraria a formação na superfície atacada de uma camada rica em sílica. $\mathrm{O}$ aumento constante de $\mathrm{OH}^{-}$em solução elevaria o $\mathrm{pH}$ local podendo favorecer uma segunda reação de quebra de ligações $\mathrm{Si}-\mathrm{O}-\mathrm{Si}$, expondo uma nova camada vítrea, provocando assim um ciclo constante de dissolução. Contudo, esse ciclo não deve ocorrer de forma agressiva nas membranas em função de seu uso se dar em um sistema não fechado, ou seja, as membranas são projetadas para operação em sistema dinâmico, aonde há uma constante passagem de fluido. Após a evidente dissolução alcalina inicial e posterior formação de superfície rica em silício, o sistema entra em equilíbrio em função do arraste de íons dissolutos, sem alteração subseqüente do pH. Desta forma, não haverá alteração significativamente da configuração de poros ou de suas características de permeabilidade.

Em suma, o processamento de elementos vítreos a partir de material reciclado, apresenta caracteristicas plenas para o emprego como produto em tecnologia avançada com certa liberdade operacional. Testes em condições reais de filtragem e avaliação das eficiencias nas diversas concentrações processadas são trabalhos em andamento cujos resultados serão publicados oportunamente.

\section{CONCLUSÕES}

Neste trabalho vimos que vidros reciclados podem ser plenamente empregados na confecção de peças porosas por sinterização. Com o uso de uma fase não sinterizável, como o $\mathrm{NaCl}$ adicionado ao pós de vidro anterior à compactação, pode-se estabelecer razoável controle sobre as características estruturais para um mesmo ciclo de aquecimento. As permeabilidades medidas nas amostras indicam, em uma primeira análise, que realmente há uma forte relação entre o teor de sal adicionado e removido e a facilidade de passagem do fluido pelo corpo poroso. Os valores mostram que adições acima de $20-25 \%$ de sal geram estrutura porosa distintas mas com permeabilidades similares. O que permite certa flexibilidade no processamento de membranas para uso específico em microfiltração.

\section{REFERÊNCIAS}

[1] W. R. Malisch, D. E. Day, B. G. Wixson, "Use of Domestic Waste Glass for Urban Paving”. National Environmental Research Center, U.S. Environmental Protection Agency, Summary Report EPA-670/2-75-053 (1975).

[2] A. C. M. Rodrigues, O. Peitl, "Reciclagem de vidros no Brasil: Um panorama geral”, in: El reciclado del vidrio em Iberoamérica. Madrid, CYTED (1999) p. 33-46

[3] R. F. Schumacher, "Monitoring and analyzing waste glass compositions”. Environ. Intern. 21, 3 (1995) 1-8.

[4] CEMPRE - Compromisso Empresarial para a Reciclagem, in: http://www.cempre.org.br, acessado em 19.09.2003

[5] http://www.ethos.org.br/sistemas/comunicacao/noticias/ vnoticias.asp?destaque $=4760$, acessado em 19.09.2003

[6] http://www.britglass.co.uk, acessado em 19.09.2003

[7] M. C. Gonçalves, R. Almeida, "Reciclagem de vidro em Portugal” in: El reciclado Del vidrio em Iberoamérica. Madrid, CYTED, 1999, p. 101-122

[8] I. W. M. Brown, K. J. D. Mackenzie, "Process design of a ceramic-like body from recycled waste glass”. J. Mater. Sci. 17 (1982) 2164-2193.

[9] Y. Lingart, "Imitation natural material tiling using waste glass”. Glass Tech. 39, 2 (1998) 42-44.

[10] M. C. Borlini, B. C. Santos, D. G. Pinatti, R. A. Conte, "Vitrificação de cerâmica vermelha usando cinza do lixo urbano" in: Congresso Brasileiro de Cerâmica, S. Paulo, SP (2002) cd-rom.

[11] R. L. Schroeder, "The Use of Recycled Materials in Highway Construction”. Public Roads 58, 2 (1994) 32-41.

[12] N. Su, J. S. Chen, "Engineering properties of asphalt concrete made with recycled glass", Resources, Conservation and Recycling 35, 4 (2002) 259-274.

[13] T. Watson, "When the Tire Hits the Glasphalt," Resource Recycling Magazine (1988) 38-41.

[14] G. Chen, H. Lee, K. L. Young, P. L. Yue, A. Wong, T. Tao, K. K. Cho, "Glass recycling in cement production-an innovative approach”. Waste Management 22, 7 (2002) 747-753. 
[15] W. Jin, C. Meyer, S. Baxter, "Glascrete - Concrete with Glass Aggregate”, ACI Mater. J. 97, 2 (2000) 208-213.

[16] Y. Shao, T. Lefort, S. Moras, D. Rodriguez, "Studies on concrete containing ground waste glass”, Cem. Conc. Res. 30, 1 (2000) 91-100.

[17] M. A. Berube, "Influence of Particle Size Distribution on the Effectiveness of Type-F fly Ash in Suppressing Expansion Due to Alkali-Silica Reactivity (SP-153)". American Concrete Institute, Publication SP153-18,153 (1995) 315-338.

[18] G. H Edwards, "Recycle of glass furnace waste materials”, J. Cleaner Production. 4, 3-4 (1996).

[19] M. Ferraris, M. Salvo, F. Smeacetto, L. Augier, L. Barbieri, A. Corradi, I. Lancellotti, "Glass matrix composites from solid waste materials”, J. Eur. Ceram. Soc. 21, 4 (2001) 453-460.

[20] F. Matteucci, M. Dondi, G. Guarini, “Effect of soda-lime glass on sintering and technological properties of porcelain stoneware tiles”, Ceram. Int. 28, 8 (2002) 873-880.

[21] Technology Brief \# GL-96-2, GL-97-10. “Two Tests of the Compatibility of Crushed Recycled glass with Plant Growth". CWC Publication, Division of the Pacific NorthWest Economic Region, Seattle, Washington, (1998) 2p.

[22] J. B. Piccirillo, R. D. Letterman, "Examination of Pulverized Waste Recycled Glass as Filter Media in Slow Sand Filtration".National Technical Information Service (NTIS) NYSERDA Report No. 97-13 Reference No. PB98158959, Albany, N.Y. (1997).

[23] S. O. Rutledge, G. A. Gagnon. "Comparing crushed recycled glass to silica sand for dual media Filtration". J. Environ. Eng. Sci. 1 (2002) 349-358.

[24] Technology Brief \# GL-95-4, "Evaluation of Crushed recycled glass as a filtration medium in slow rate filtration".

CWC Publication, Division of the Pacific NorthWest Economic Region, Seattle, Washington, (1997) 2p.

[25] O. B. G. Assis, L. C. Claro, "Processing of Soda Lime Glass Membranes by Filler Principle for the Enzyme Coupling Application”. J. Non-Cryst. Sol. 247 (1999) 237240.

[26] O. B. G. Assis, M. Ferrante, D. C. Vieira, "Open pore microfiltration membranes produced from waste glass". Glass Technology 42, 3 (2001) 101-103.

[27] G. Divino, O. B. G. Assis, D. C. Vieira, "Desenvolvimento de Filtros de Baixo Custo de Interesse à Agroindústria”. in Anais do II Simpósio Nacional de Instrumentação Agropecuária, S. Carlos, SP (1998) p. 328-334

[28] Filtro Inovador na Agricultura, Revista Pesquisa FAPESP 71 (2002) 68-69.
[29] J. M. Naime, O. B. G. Assis, G. Divino, C. M. P. Vaz, A. Macedo, "Analisador Granulométrico Automatizado Aplicado na Caracterização de Pós de Vidros Processados por Moagem". in Anais do II Simpósio Nacional de Instrumentação Agropecuária, S. Carlos, SP (1998) 257260.

[30] C. M. P. Vaz, J. M. Naime, A. Macedo, "Soil particle fraction determined by gamma-ray attenuation". Soil Sci. 164, 6 (1999) 403-410.

[31] F. B. Siebers, N. Greiulich, W., Kiefer, "Manufacture, Properties and Application of open-pore Sintered Glasses and open-pore sintered glass-ceramics”. Glastech. Ber. 62, 2 (1989) 63-73

[32] R. A. Vasques, M. D. M. Innocentini, O. B. G. Assis, “A Simple apparatus for determining the permeability of thinthickness porous materials by pressure-decay Technique". Rev. Fís. Apl. Instrum. 14, 4 (1999) 120-123.

[33] R. A. Vasques, "Preparação e caracterização de elementos vítreos porosos para usos em microfiltração". Dissertação de Mestrado, Interunidades Ciência e Engenharia de Materiais - USP, S. Carlos, SP (2001) 117p.

[34] M. D. M. Innocentini, V. R. Salvini, V. C. Pandolfelli, "How accurate is Darcy's law for refractories". Am. Ceram. Soc. Bull. 77, 11 (1999) 64-68.

[35] G. W. Scherer, "Sintering of Low-density Glasses: I, Theory”. J. Am. Ceram. Soc. 60, 5-6 (1976) 236-239.

[36] Y. Chiang, D. Birnie III, W. D. Kingery "Physical Ceramics”. John Wiley and Sons, Inc., N. York (1977) p. 392-398.

[37] J. Zarycki, “Glasses and the vitreous state”. Cambridge University Press, Cambridge (1991) 505p.

[38] D. C. Vieira, "Fabricação de elementos vítreos porosos para o depósito de polímeros visando a obtenção de membranas com superfícies ativas”. Tese de Doutoramento, Interunidades Ciência e Engenharia de Materiais - USP., S. Carlos, SP (2001) 146p.

[39] J. F. Thovert, J. Salles, P. M. Adler. "Computerized characterization of the geometry of real porous media: their discretization, analysis and interpretation, J. Microsc. 170 (1993) 65-79.

[40] R. A. Vasques, M. D. M. Innocentini, O. B. G. Assis, "Porosidade e permeabilidade de membranas vítreas obtidas de material reciclado". in Anais do $45^{\circ}$ Congresso Brasileiro de Cerâmica, Joinvile, SC (2001) p. 401-408, cd rom.

[41] C. W. Sinton, W. C. LaCourse. "Experimental survey of the chemical durability of commercial soda-lime-silicate glasses”. Mater. Res. Bull. 36 (2001) 2471-2479

(Rec. 24/09/2003, Rev. 02/03/04-07/02/2006, Ac.17/02/2006) 